MIFP-06-10

hep-th/0604125

April 2006

\title{
General Kerr-NUT-AdS Metrics in All Dimensions
}

\author{
W. Chen, H. Lü and C.N. Pope \\ ${ }^{\dagger}$ George P. $\&$ Cynthia W. Mitchell Institute for Fundamental Physics, \\ Texas A EM University, College Station, TX 77843-4242, USA
}

\begin{abstract}
$\underline{\text { ABSTRACT }}$
The Kerr-AdS metric in dimension $D$ has cohomogeneity $[D / 2]$; the metric components depend on the radial coordinate $r$ and $[D / 2]$ latitude variables $\mu_{i}$ that are subject to the constraint $\sum_{i} \mu_{i}^{2}=1$. We find a coordinate reparameterisation in which the $\mu_{i}$ variables are replaced by $[D / 2]-1$ unconstrained coordinates $y_{\alpha}$, and having the remarkable property that the Kerr-AdS metric becomes diagonal in the coordinate differentials $d y_{\alpha}$. The coordinates $r$ and $y_{\alpha}$ now appear in a very symmetrical way in the metric, leading to an immediate generalisation in which we can introduce $[D / 2]-1$ NUT parameters. We find that $(D-5) / 2$ are non-trivial in odd dimensions, whilst $(D-2) / 2$ are non-trivial in even dimensions. This gives the most general Kerr-NUT-AdS metric in $D$ dimensions. We find that in all dimensions $D \geq 4$ there exist discrete symmetries that involve inverting a rotation parameter through the AdS radius. These symmetries imply that Kerr-NUT-AdS metrics with over-rotating parameters are equivalent to under-rotating metrics. We also consider the BPS limit of the Kerr-NUT-AdS metrics, and thereby obtain, in odd dimensions and after Euclideanisation, new families of Einstein-Sasaki metrics.
\end{abstract}

Research supported in part by DOE grant DE-FG03-95ER40917. 


\section{Contents}

1 Introduction 2

2 The General Kerr-NUT-AdS Solutions 6

2.1 The odd-dimensional case: $D=2 n+1 \ldots \ldots \ldots \ldots$

2.2 The even-dimensional case: $D=2 n \ldots \ldots \ldots \ldots$

3 A Simpler Form for the Kerr-NUT-AdS Metrics $\quad 8$

$3.1 \quad D=2 n+1$ dimensions . . . . . . . . . . . . . . . . . . . . 9

$3.2 \quad D=2 n$ dimensions . . . . . . . . . . . . . . . . . 11

4 Kerr-NUT-AdS Metrics in $D=6$ and $D=7 \quad 14$

4.1 Seven-dimensional Kerr-NUT-AdS . . . . . . . . . . . . . . . . . . . . 14

4.2 Six-dimensional Kerr-NUT-AdS . . . . . . . . . . . . . . . . . . . 16

5 BPS Limits $\quad 17$

5.1 BPS limit for $D=2 n+1 \ldots \ldots \ldots \ldots \ldots \ldots$

$5.2 \quad$ BPS limit for $D=2 n \ldots \ldots \ldots \ldots \ldots \ldots \ldots$

6 Conclusions 20

A A symmetry between the time and azimuthal coordinates 22

B Inversion symmetry of the $D=4$ rotating black hole 22

\section{Introduction}

With the discovery of higher-dimensional supergravities, string theory and M-theory, it has been appreciated that higher-dimensional solutions of the Einstein and supergravity equations have an important rôle to play. With this realisation, it becomes of considerable interest to carry out investigations in higher dimensions, generalising the earlier very extensive four-dimensional studies, of the most general classes of solutions subject to certain symmetry assumptions. In this regard, black hole metrics and their generalisations are amongst the most important solutions worthy of investigation.

In four dimensions the early discovery of Schwarzschild solution was quickly followed by the extension to the Reissner-Nordström solution, and, considerably later, by the discovery 
of generalisations with rotation, a cosmological constant, a NUT parameter, and an acceleration parameter. The general solution containing all these parameters was obtained in [1]. Less is known in higher dimensions, and it was not until 1986 [2] that the generalisation of the Kerr solution to dimensions $D \geq 5$ was obtained. A feature of these higher-dimensional black holes is that there are $[(D-1) / 2]$ independent rotation parameters, corresponding to independent rotations in the $[(D-1) / 2]$ orthogonal spatial 2-planes. The metrics in general have cohomogeneity $[D / 2]$ in $D$ dimensions, with lower cohomogeneities arising in specialisations where some of the rotation parameters are set equal. The generalisation to include a cosmological constant was obtained in five dimensions in [3], and in arbitrary dimension in $[4,5]$.

It is of interest to generalise these higher-dimensional Kerr-AdS metrics further, by including also NUT charge. Recent progress in this direction was made in [6], where it was shown that one can introduce a NUT charge parameter in all the Kerr-AdS metrics in $D \geq 6$ if they are first specialised, by equating rotation parameters appropriately, to have cohomogeneity 2 . The case of $D=4$ had, of course, been obtained long ago, and the case $D=5$ turns out to be rather degenerate, in that the NUT parameter is trivial and can be removed by a redefinition of the other parameters and the coordinates. (Cohomogeneity-one pure multi-nut solutions in higher dimensions were obtained in [7].)

The purpose of the present paper is to present new NUT generalisations of the KerrAdS metrics which are, we believe, of the most general possible type. We find that in $D$ dimensions the general Kerr-AdS metric (with all rotation parameters allowed to be unequal) can be extended by the inclusion of $(D-5) / 2$ independent NUT parameters when $D$ is odd, and $(D-2) / 2$ when $D$ is even. We arrived at these solutions by first rewriting the Kerr-AdS metrics using a set of coordinate variables that make the introduction of the NUT parameters a very natural generalisation of the usual mass parameter.

The choice of coordinates in four dimensions that leads to the natural inclusion of a NUT parameter in the Kerr-AdS solution is rather well known. In the standard description of the Kerr-AdS solution one has angular coordinates $(\theta, \phi)$ parameterising the 2-sphere spatial sections at constant radius $r$. If one defines $y=a \cos \theta$, where $a$ is the rotation parameter, and makes appropriate linear redefinitions of the time and azimuthal coordinate $\phi$, the metric can be written as

$$
d s_{4}^{2}=-\frac{\Delta_{r}}{r^{2}+u^{2}}\left(d \tau+y^{2} d \psi\right)^{2}+\frac{\Delta_{y}}{r^{2}+u^{2}}\left(d \tau-r^{2} d \psi\right)^{2}+\frac{\left(r^{2}+y^{2}\right) d r^{2}}{\Delta_{r}}+\frac{\left(r^{2}+y^{2}\right) d y^{2}}{\Delta_{y}},
$$

where

$$
\Delta_{r}=\left(r^{2}+a^{2}\right)\left(1+g^{2} r^{2}\right)-2 M r, \quad \Delta_{y}=\left(a^{2}-y^{2}\right)\left(1-g^{2} y^{2}\right) .
$$


This Kerr-AdS solution, satisfying $R_{\mu \nu}=-3 g^{2} g_{\mu \nu}$, is generalised to include the NUT parameter $L$ by replacing $\Delta_{y}$ by

$$
\Delta_{y}=\left(a^{2}-y^{2}\right)\left(1-g^{2} y^{2}\right)+2 L y .
$$

An important feature of this parameterisation, which makes the inclusion of the NUT parameter very natural, is that the radial variable $r$ and the "latitude" variable $y$ are placed on a very symmetrical footing. The NUT generalisations of the higher-dimensional KerrAdS metrics that were obtained in [6] worked in a very similar way. An essential part of the construction was that the rotation parameters had to be specialised in such a way that the cohomogeneity was reduced to 2 , and so again a latitude-type coordinate $y$ could be introduced in such a way that it, and the radial variable $r$, appeared in a very symmetrical way. The metric functions depended on $r$ and $y$, with the $(D-2)$-dimensional hypersurfaces at constant $r$ and $y$ being homogeneous.

The key to finding the NUT generalisations that we obtain in the present paper is to make a suitable reparameterisation of the multiple "latitude" coordinates that arise in the higher-dimensional Kerr-AdS metrics. In $D$ dimensions one has the time and radial variables $(t, r),[(D-1) / 2]$ azimuthal angles $\phi_{i}$ and $[D / 2]$ latitude, or direction cosine, coordinates $\mu_{i}$, which are subject to the constraint

$$
\sum_{i=1}^{[D / 2]} \mu_{i}^{2}=1
$$

The spatial sections at constant radius $r$ have the geometry of deformed $(D-2)$-spheres. The unit $S^{D-2}$ metric is given by

$$
d \Omega^{2}=\sum_{i=1}^{[D / 2]} d \mu_{i}^{2}+\sum_{i=1}^{[(D-1) / 2]} \mu_{i}^{2} d \phi_{i}^{2}
$$

in these variables. Associated with each azimuthal angle $\phi_{i}$ is a rotation parameter $a_{i}$.

We find that the appropriate reparameterisation of the $\mu_{i}$ coordinates is as follows. Taking $D=2 n+1$ in the odd-dimensional case, and $D=2 n$ in the even-dimensional case, we parameterise the $n$ coordinates $\mu_{i}$ as

$$
\mu_{i}^{2}=\frac{\prod_{\alpha=1}^{n-1}\left(a_{i}^{2}-y_{\alpha}^{2}\right)}{\prod_{k=1}^{\prime n}\left(a_{i}^{2}-a_{k}^{2}\right)},
$$

where the prime on $\prod^{\prime}$ indicates that the term that vanishes (i.e. when $k=i$ ) is omitted from the product. ${ }^{1}$ Note that this parameterisation using just $(n-1)$ coordinates $y_{\alpha}$ explicitly

\footnotetext{
${ }^{1}$ Transformations of this type were first considered by Jacobi, in the context of constrained dynamical systems [8].
} 
solves the constraint (4). It also has the striking property that it diagonalises the metric (5) on the unit sphere, expressed in terms of the unconstrained latitude variables $y_{\alpha}$ :

$$
d \Omega^{2}=\sum_{\alpha=1}^{n-1} g_{\alpha} d y_{\alpha}^{2}+\sum_{i=1}^{[(D-1) / 2]} \mu_{i}^{2} d \phi_{i}^{2},
$$

where the notation $\prod^{\prime}$ universally indicates that the vanishing factor is to be omitted from the product, $\mu_{i}^{2}$ is given by(6), and

$$
g_{\alpha}=-\frac{y_{\alpha}^{2} \prod_{\beta=1}^{\prime n-1}\left(y_{\alpha}^{2}-y_{\beta}^{2}\right)}{\prod_{k=1}^{n}\left(a_{k}^{2}-y_{\alpha}^{2}\right)} .
$$

Note that the parameterisation (6) solves the constraint (4), and diagonalises the metric as in (7), for arbitrary choices of unequal constants $a_{i}^{2}$.

When we utilise (6) in the next section, we shall take the constants $a_{i}$ to be the rotation parameters of the Kerr-AdS black holes. In the case of even dimensions $D=2 n$, there are only $(n-1)$ rotation parameters, and so $a_{n}$ is taken to be zero. We shall see that with this choice of the parameters in the Jacobi transformations (6), the Kerr-AdS metrics obtained in $[4,5]$, which are non-diagonal in the latitude coordinate differentials $d \mu_{i}$, remarkably become diagonal with respect to the unconstrained coordinate differentials $d y_{\alpha}$. Furthermore, we shall see that after writing the Kerr-AdS metrics in terms of the coordinates $\left(t, r, y_{\alpha}, \phi_{i}\right)$, the radial variable $r$ and the latitude variables $y_{\alpha}$ enter the metrics in a very symmetrical fashion, such that the generalisation to include a set of $(n-1)$ NUT parameters becomes very natural. We have explicitly verified, with the aid of a computer, that in all dimensions $D \leq 15$ these generalisations of the Kerr-AdS metrics satisfy the Einstein equations. Since there are no special features peculiar to these dimensions, we can with confidence expect that the generalisations satisfy the Einstein equations in all dimensions.

After presenting the general Kerr-NUT-AdS metrics in section 2, we then consider, in section 3, some simpler expressions for the Kerr-NUT-AdS metrics. It turns out that the symmetrical appearance of the radial and latitude variables is further enhanced if one performs a "Wick rotation" of the radial coordinate $r$, and defines variables $x_{\mu}$ with $x_{\alpha}=y_{\alpha}$, $x_{n}=\mathrm{i} r$. This leads to a form for the metric in which all the coordinates $x_{\mu}$ enter on an exactly parallel footing. In a further simplification of the expressions for the metrics, we find that by defining appropriate linear combinations of the time and azimuthal coordinates, the Kerr-NUT-AdS metrics can be cast in a form that provides a natural generalisation of the four-dimensional metrics described in [9]. We also discuss certain scaling symmetries and discrete symmetries of the Kerr-NUT-AdS metrics. The scaling symmetries imply that 
there are $(n-2)$ non-trivial NUT parameters in odd dimensions $D=2 n+1$, and $(n-1)$ non-trivial NUT parameters in even dimensions $D=2 n$. The discrete symmetries imply that metrics with over-rotation, i.e. where one or more rotation parameters exceeds the AdS radius, are equivalent to metrics with under-rotation.

In section 4 , we focus on the particular cases of dimensions $D=6$ and $D=7$, since these are the lowest dimensions where our new results extend beyond those known previously. In section 5 we study the supersymmetric, or BPS, limits of the new metrics in odd and even dimensions. After performing a Euclideanisation, the odd-dimensional solutions give rise to new examples of Einstein-Sasaki metrics in $D \geq 7$. By writing these as circle fibrations over an Einstein-Kähler base, we thereby obtain new classes of Einstein-Kähler metrics in all even dimensions $D \geq 6$. The paper ends with conclusions in section 6 .

\section{The General Kerr-NUT-AdS Solutions}

In this section, we shall present our general results for the Kerr-NUT-AdS metrics in $D$ dimensions. These ostensibly have a total of $(D-1)$ independent parameters, comprising the mass $M$, the $[(D-1) / 2]$ rotation parameters $a_{i}$, and $[(D-2) / 2]$ NUT parameters $L_{\alpha}$. As we shall discuss later, in odd dimensions there is a symmetry that allows one to eliminate one of the parameters, and so in odd dimensions there are actually in total $(D-2)$ non-trivial parameters in the solutions we obtain.

The first step is to rewrite the Kerr-AdS metrics, which were obtained in [4, 5], in terms of the new coordinate parameterisation introduced in (6). It is advantageous to separate the discussion into two cases, depending upon whether $D$ is odd or even.

\subsection{The odd-dimensional case: $D=2 n+1$}

As a preliminary, we make the following definitions:

$$
\begin{aligned}
U & =\prod_{\alpha=1}^{n-1}\left(r^{2}+y_{\alpha}^{2}\right), \quad U_{\alpha}=-\left(r^{2}+y_{\alpha}^{2}\right) \prod_{\beta=1}^{\prime n-1}\left(y_{\beta}^{2}-y_{\alpha}^{2}\right), \quad 1 \leq \alpha \leq n-1, \\
W & =\prod_{\alpha=1}^{n-1}\left(1-g^{2} y_{\alpha}^{2}\right), \quad \gamma_{i}=\prod_{\alpha=1}^{n-1}\left(a_{i}^{2}-y_{\alpha}^{2}\right), \quad 1 \leq i \leq n \\
X & =\frac{1+g^{2} r^{2}}{r^{2}} \prod_{k=1}^{n}\left(r^{2}+a_{k}^{2}\right)-2 M \\
X_{\alpha} & =\frac{1-g^{2} y_{\alpha}^{2}}{y_{\alpha}^{2}} \prod_{k=1}^{n}\left(a_{k}^{2}-y_{\alpha}^{2}\right)+2 L_{\alpha} \quad 1 \leq \alpha \leq n-1 .
\end{aligned}
$$


We have actually already included the new NUT parameters $L_{\alpha}$ here; they appear just in the definitions of the functions $X_{\alpha}$. Note that again the notation $\prod^{\prime}$ indicates that the term in the full product that vanishes is to be omitted.

Using these functions, we find that the Kerr-NUT-AdS metrics in $D=2 n+1$ dimensions are given by

$$
\begin{aligned}
d s^{2}= & \frac{U}{X} d r^{2}+\sum_{\alpha=1}^{n-1} \frac{U_{\alpha}}{X_{\alpha}} d y_{\alpha}^{2}-\frac{X}{U}\left[W d \tilde{t}-\sum_{i=1}^{n} a_{i}^{2} \gamma_{i} d \tilde{\phi}_{i}\right]^{2} \\
& +\sum_{\alpha=1}^{n-1} \frac{X_{\alpha}}{U_{\alpha}}\left[\frac{\left(1+g^{2} r^{2}\right) W}{1-g^{2} y_{\alpha}^{2}} d \tilde{t}-\sum_{i=1}^{n} \frac{a_{i}^{2}\left(r^{2}+a_{i}^{2}\right) \gamma_{i}}{a_{i}^{2}-y_{\alpha}^{2}} d \tilde{\phi}_{i}\right]^{2} \\
& +\frac{\prod_{k=1}^{n} a_{k}^{2}}{r^{2} \prod_{\alpha=1}^{n-1} y_{\alpha}^{2}}\left[\left(1+g^{2} r^{2}\right) W d \tilde{t}-\sum_{i=1}^{n}\left(r^{2}+a_{i}^{2}\right) \gamma_{i} d \tilde{\phi}_{i}\right]^{2} .
\end{aligned}
$$

With the parameters $L_{\alpha}$ set to zero, the metrics are just a rewriting of the Kerr-AdS metrics obtained in $[4,5]$, using the new coordinates $y_{\alpha}$ defined by $(6) .^{2}$ They are written here in an asymptotically-static frame. We have also rescaled the time and azimuthal coordinates in order to simplify the expression. They are related to the original asymptotically static coordinates $\left(t, \phi_{i}\right)$ by

$$
t=\tilde{t} \prod_{i=1}^{n} \Xi_{i}, \quad \phi_{i}=a_{i} \Xi_{i} \tilde{\phi}_{i} \prod_{k=1}^{\prime n}\left(a_{i}^{2}-a_{k}^{2}\right),
$$

where $\Xi_{i}=1-g^{2} a_{i}^{2}$. The coordinate $t$ is canonically normalised, and the coordinates $\phi_{i}$ each have period $2 \pi$, in the Kerr-AdS metrics.

The new metrics that we have obtained, by including the $(n-1)$ parameters $L_{\alpha}$ in the definition of $X_{\alpha}$ in (9), describe the general Kerr-NUT-AdS metrics in dimension $D=2 n+1$. As we shall discuss in section 3.1, in odd dimensions there is actually a redundancy among the $(n-1)$ NUT parameters, with one of them being trivial. Thus the total count of nontrivial parameters in the general Kerr-NUT-AdS metrics in dimension $D=2 n+1$ is $2 n-1$, which can be thought of $n$ rotation parameters, the mass, and $(n-2)$ NUT charges.

\subsection{The even-dimensional case: $D=2 n$}

In this case we begin by defining functions as follows:

$$
U=\prod_{\alpha=1}^{n-1}\left(r^{2}+y_{\alpha}^{2}\right), \quad U_{\alpha}=-\left(r^{2}+y_{\alpha}^{2}\right) \prod_{\beta=1}^{\prime n-1}\left(y_{\beta}^{2}-y_{\alpha}^{2}\right), \quad 1 \leq \alpha \leq n-1,
$$

\footnotetext{
${ }^{2}$ Note that the metric signature is just the usual $(-++\cdots+)$, for the appropriate choices of the $y_{\alpha}$ coordinate intervals that correspond to the standard Kerr-AdS black hole solution.
} 


$$
\begin{aligned}
W & =\prod_{\alpha=1}^{n-1}\left(1-g^{2} y_{\alpha}^{2}\right), \quad \gamma_{i}=\prod_{\alpha=1}^{n-1}\left(a_{i}^{2}-y_{\alpha}^{2}\right), \quad 1 \leq i \leq n-1, \\
X & =\left(1+g^{2} r^{2}\right) \prod_{k=1}^{n-1}\left(r^{2}+a_{k}^{2}\right)-2 M r, \\
X_{\alpha} & =-\left(1-g^{2} y_{\alpha}^{2}\right) \prod_{k=1}^{n-1}\left(a_{k}^{2}-y_{\alpha}^{2}\right)-2 L_{\alpha} y_{\alpha}, \quad 1 \leq \alpha \leq n-1 .
\end{aligned}
$$

We find that the Kerr-NUT-AdS metrics in $D=2 n$ dimensions are given by

$$
\begin{aligned}
d s^{2}= & \frac{U}{X} d r^{2}+\sum_{\alpha=1}^{n-1} \frac{U_{\alpha}}{X_{\alpha}} d y_{\alpha}^{2}-\frac{X}{U}\left[W d \tilde{t}-\sum_{i=1}^{n-1} \gamma_{i} d \tilde{\phi}_{i}\right]^{2} \\
& +\sum_{\alpha=1}^{n-1} \frac{X_{\alpha}}{U_{\alpha}}\left[\frac{\left(1+g^{2} r^{2}\right) W}{1-g^{2} y_{\alpha}^{2}} d \tilde{t}-\sum_{i=1}^{n-1} \frac{\left(r^{2}+a_{i}^{2}\right) \gamma_{i}}{a_{i}^{2}-y_{\alpha}^{2}} d \tilde{\phi}_{i}\right]^{2} .
\end{aligned}
$$

Again, the previously-known Kerr-AdS metrics correspond to setting the new NUT parameters $L_{\alpha}$ to zero in the definition of the functions $X_{\alpha}$ in (12). The coordinates $\tilde{t}$ and $\tilde{\phi}_{i}$ are related to the canonically-normalised coordinates $t$ and $\phi_{i}$ of the $L_{\alpha}=0$ Kerr-AdS metrics by

$$
t=\tilde{t} \prod_{i=1}^{n} \Xi_{i}, \quad \phi_{i}=a_{i} \Xi_{i} \tilde{\phi}_{i} \prod_{k=1}^{\prime n-1}\left(a_{i}^{2}-a_{k}^{2}\right),
$$

When $L_{\alpha}=0$, regularity of the Kerr-AdS metric dictates that the azimuthal angles $\phi_{i}$ should all have period $2 \pi$.

As we shall discuss in section 3.2, all the NUT parameters are non-trivial in even dimensions, and so the general Kerr-NUT-AdS metrics in dimension $D=2 n$ have $2 n-1$ independent parameters, comprising $(n-1)$ rotations, the mass, and $(n-1)$ NUT parameters.

\section{A Simpler Form for the Kerr-NUT-AdS Metrics}

We already saw in section 2 that the Kerr-NUT-AdS metrics assume a rather symmetrical form when the latitude coordinates $\mu_{i}$ are parameterised in terms of the coordinates $y_{\alpha}$ using (6). The parallel between the radial coordinate $r$ and the latitude coordinates $y_{\alpha}$ becomes even more striking if we perform a Wick rotation of the radial variable, and define the $n$ coordinates $x_{\mu}$ by

$$
x_{n}=\mathrm{i} r, \quad x_{\alpha}=y_{\alpha}, \quad 1 \leq \alpha \leq n-1 .
$$

As we shall show, the Kerr-NUT-AdS metrics can now be written in a considerably simpler form. In fact, if we then perform further transformations on the time and azimuthal coor- 
dinates, we arrive at an even simpler way of presenting the Kerr-NUT-AdS metrics, which generalises the four-dimensional expressions obtained in [9]. As always, it is convenient to separate the discussion at this stage into the cases of odd and even dimensions.

\section{1 $D=2 n+1$ dimensions}

We first define the functions

$$
\begin{aligned}
& U_{\mu}=\prod_{\nu=1}^{\prime n}\left(x_{\nu}^{2}-x_{\mu}^{2}\right), \quad X_{\mu}=\frac{\left(1-g^{2} x_{\mu}^{2}\right)}{x_{\mu}^{2}} \prod_{k=1}^{n}\left(a_{k}^{2}-x_{\mu}^{2}\right)+2 M_{\mu}, \\
& \widetilde{W}=\prod_{\nu=1}^{n}\left(1-g^{2} x_{\nu}^{2}\right), \quad \tilde{\gamma}_{i}=\prod_{\nu=1}^{n}\left(a_{i}^{2}-x_{\nu}^{2}\right) .
\end{aligned}
$$

The odd-dimensional Kerr-NUT-AdS metric (10) can then be written as

$$
\begin{aligned}
d s^{2}= & \sum_{\mu=1}^{n}\left\{\frac{U_{\mu}}{X_{\mu}} d x_{\mu}^{2}+\frac{X_{\mu}}{U_{\mu}}\left[\frac{\widetilde{W}}{1-g^{2} x_{\mu}^{2}} d \tilde{t}-\sum_{i=1}^{n} \frac{a_{i}^{2} \tilde{\gamma}_{i}}{a_{i}^{2}-x_{\mu}^{2}} d \tilde{\phi}_{i}\right]^{2}\right\} \\
& -\frac{\prod_{k=1}^{n} a_{k}^{2}}{\prod_{\mu=1}^{n} x_{\mu}^{2}}\left[\widetilde{W} d \tilde{t}-\sum_{i=1}^{n} \tilde{\gamma}_{i} d \tilde{\phi}_{i}\right]^{2} .
\end{aligned}
$$

Note that $M_{n}$ is just equal to the previous mass parameter $M$, while the remaining $M_{\alpha}$ are NUT parameters, previously denoted by $L_{\alpha}$.

It is useful to give also the inverse of the metric (17). Defining

$$
S_{\mu}=\prod_{k=1}^{n}\left(a_{k}^{2}-x_{\mu}^{2}\right)^{2}, \quad B_{j}=\prod_{k=1}^{\prime n}\left(a_{j}^{2}-a_{k}^{2}\right),
$$

we find that the inverse metric is given by

$$
\begin{aligned}
\left(\frac{\partial}{\partial s}\right)^{2}= & \sum_{\mu=1}^{n}\left\{\frac{X_{\mu}}{U_{\mu}}\left(\frac{\partial}{\partial x_{\mu}}\right)^{2}+\frac{S_{\mu}}{x_{\mu}^{4} U_{\mu} X_{\mu}}\left[\frac{1}{\left(\prod_{j} \Xi_{j}\right)} \frac{\partial}{\partial \tilde{t}}+\sum_{k=1}^{n} \frac{\left(1-g^{2} x_{\mu}^{2}\right)}{B_{k} \Xi_{k}\left(a_{k}^{2}-x_{\mu}^{2}\right)} \frac{\partial}{\partial \tilde{\phi}_{k}}\right]^{2}\right\} \\
& -\frac{\left(\prod_{k=1}^{n} a_{k}^{2}\right)}{\left(\prod_{\nu=1}^{n} x_{\nu}^{2}\right)}\left(\frac{1}{\left(\prod_{j} \Xi_{j}\right)} \frac{\partial}{\partial \tilde{t}}+\sum_{k=1}^{n} \frac{1}{a_{k}^{2} B_{k} \Xi_{k}} \frac{\partial}{\partial \tilde{\phi}_{k}}\right)^{2} .
\end{aligned}
$$

The inverse metric becomes somewhat simpler if expressed in terms of the original canon-

ically normalised coordinates $t$ and $\phi_{k}$, whose relation to $\tilde{t}$ and $\tilde{\phi}_{k}$ is given in (11). The metric (19) then becomes

$$
\begin{aligned}
\left(\frac{\partial}{\partial s}\right)^{2}= & \sum_{\mu=1}^{n}\left\{\frac{X_{\mu}}{U_{\mu}}\left(\frac{\partial}{\partial x_{\mu}}\right)^{2}+\frac{S_{\mu}}{x_{\mu}^{4} U_{\mu} X_{\mu}}\left[\frac{\partial}{\partial t}+\sum_{k=1}^{n} \frac{a_{k}\left(1-g^{2} x_{\mu}^{2}\right)}{\left(a_{k}^{2}-x_{\mu}^{2}\right)} \frac{\partial}{\partial \phi_{k}}\right]^{2}\right\} \\
& -\frac{\left(\prod_{k=1}^{n} a_{k}^{2}\right)}{\left(\prod_{\nu=1}^{n} x_{\nu}^{2}\right)}\left(\frac{\partial}{\partial t}+\sum_{k=1}^{n} \frac{1}{a_{k}} \frac{\partial}{\partial \phi_{k}}\right)^{2} .
\end{aligned}
$$


It is straightforward to see that the Kerr-NUT-AdS metrics (10) and (17) have a set of discrete symmetries under which one of the rotation parameters $a_{i}$ is inverted through the AdS radius $1 / g$. Thus, choosing $a_{1}$ for this purpose as a representative example, we can see that (17) is invariant under the set of transformations

$$
\begin{aligned}
& a_{1} g \rightarrow \frac{1}{a_{1} g}, \quad a_{j} \rightarrow \frac{a_{j}}{a_{1} g}, \quad 2 \leq j \leq n, \\
& M_{\mu} \rightarrow \frac{M_{\mu}}{\left(a_{1} g\right)^{2 n}}, \quad g t \rightarrow \phi_{1}, \quad \phi_{1} \rightarrow g t, \quad x_{\mu} \rightarrow \frac{x_{\mu}}{a_{1} g},
\end{aligned}
$$

with $\phi_{j}$ for $2 \leq j \leq n$ left unchanged. This, and the other permutation-related inversion symmetries, can always map a metric with over-rotation (one or more parameters $a_{i}$ satisfying $\left|a_{i} g\right|>1$ ) into a metric with under-rotation (all parameters satisfying $\left|a_{i} g\right|<1$ ).

A further simplification of the new Kerr-NUT-AdS metrics that we obtained in (10) and (17) in dimensions $D=2 n+1$ is possible, allowing them to be written in a manner that is a rather natural higher-dimensional analogue of the expression in [9] for the four-dimensional rotating black hole metrics. Again we begin by performing the "Wick rotation" of the radial variable, as in (15). We then find that after appropriate linear redefinitions of the time and azimuthal coordinates, the $D=2 n+1$ Kerr-NUT-AdS metrics can be written as

$$
d s^{2}=\sum_{\mu=1}^{n}\left\{\frac{d x_{\mu}^{2}}{Q_{\mu}}+Q_{\mu}\left(\sum_{k=0}^{n-1} A_{\mu}^{(k)} d \psi_{k}\right)^{2}\right\}-\frac{c}{\left(\prod_{\nu=1}^{n} x_{\nu}^{2}\right)}\left(\sum_{k=0}^{n} A^{(k)} d \psi_{k}\right)^{2}
$$

where we define

$$
\begin{aligned}
Q_{\mu} & =\frac{X_{\mu}}{U_{\mu}}, \quad U_{\mu}=\prod_{\nu=1}^{\prime n}\left(x_{\nu}^{2}-x_{\mu}^{2}\right), \quad X_{\mu}=\sum_{k=1}^{n} c_{k} x_{\mu}^{2 k}+\frac{c}{x_{\mu}^{2}}-2 b_{\mu}, \\
A_{\mu}^{(k)} & =\sum_{\nu_{1}<\nu_{2}<\cdots<\nu_{k}}^{\prime} x_{\nu_{1}}^{2} x_{\nu_{2}}^{2} \cdots x_{\nu_{k}}^{2}, \quad A^{(k)}=\sum_{\nu_{1}<\nu_{2} \cdots<\nu_{k}} x_{\nu_{1}}^{2} x_{\nu_{2}}^{2} \cdots x_{\nu_{k}}^{2} .
\end{aligned}
$$

Here, the prime on the summation symbol in the definition of $A_{\mu}^{k}$ indicates that the index value $\mu$ is omitted in the summations of the $\nu$ indices over the range $[1, n]$. Note that $\psi_{0}$ plays the rôle of the time coordinate. It is worth remarking that $A^{(k)}$ and $A_{\mu}^{(k)}$ can be defined via the generating functions

$$
\prod_{\nu=1}^{n}\left(1+\lambda x_{\nu}^{2}\right)=\sum_{k=0}^{n} \lambda^{k} A^{(k)}, \quad\left(1+\lambda x_{\mu}^{2}\right)^{-1} \prod_{\nu=1}^{n}\left(1+\lambda x_{\nu}^{2}\right)=\sum_{k=0}^{n-1} \lambda^{k} A_{\mu}^{(k)} .
$$

The constants $c_{k}, c$ and $b_{\mu}$ are arbitrary, with $c_{n}=(-1)^{n+1} g^{2}$ determining the value of the cosmological constant, $R_{\mu \nu}=-2 n g^{2} g_{\mu \nu}$. The remaining $2 n$ constants $c_{k}, c$ and $b_{\mu}$ are related to the $n$ rotation parameters $a_{i}$, the mass $M$ and the $(n-1)$ NUT parameters 
$L_{\alpha}$ in the obvious way that follows by comparing $X_{\mu}$ in (23) with $X_{\mu}$ in (16). However, it should be noted that not all the parameters are non-trivial in the general solution. This can be seen from the fact that there is a scaling symmetry of the metric (22), under which we send

$$
\begin{aligned}
& x_{\mu} \rightarrow \lambda x_{\mu}, \quad \psi_{k} \rightarrow \lambda^{-2 k-1} \psi_{k}, \\
& c_{k} \rightarrow \lambda^{2 n-2 k} c_{k}, \quad c \rightarrow \lambda^{2 n+2} c, \quad b_{\mu} \rightarrow \lambda^{2 n} b_{\mu} .
\end{aligned}
$$

This scaling symmetry implies that there is one trivial parameter in the general Kerr-NUTAdS solution, leaving a total of $2 n-1$ non-trivial parameters in $D=2 n+1$ dimensions. In fact, in odd dimensions there is not necessarily a clear distinction between rotation parameters and NUT parameters, as can be seen by comparing the expressions for the functions $X_{\mu}$ in (23), and the expressions in terms of rotations, mass and NUT parameters in (16). One might for example find that for some values of the parameters, if the scaling symmetry (25) is used in order to remove a "redundant" NUT charge, then this leads to a rotation parameter becoming imaginary. In such a range of the parameters, it would be more natural to retain the redundant NUT parameter. This is quite different from the situation in even dimensions, where the mass and NUT parameters are distinguished by being the coefficients of linear powers of the coordinates, as can be seen in (12) and in (27) below.

We find that the inverse of the metric (22) is given by

$$
\begin{aligned}
\left(\frac{\partial}{\partial s}\right)^{2}= & \sum_{\mu=1}^{n}\left\{Q_{\mu}\left(\frac{\partial}{\partial x_{\mu}}\right)^{2}+\frac{1}{x_{\mu}^{4} Q_{\mu} U_{\mu}^{2}}\left[\sum_{k=0}^{n}(-1)^{k} x_{\mu}^{2(n-k)} \frac{\partial}{\partial \psi_{k}}\right]^{2}\right\} \\
& -\frac{1}{c\left(\prod_{\nu=1}^{n} x_{\nu}^{2}\right)}\left(\frac{\partial}{\partial \psi_{n}}\right)^{2} .
\end{aligned}
$$

The specific case of the Kerr-NUT-AdS metric in $D=7$ dimensions is discussed in section 4.1, including the explicit transformation of the time and azimuthal coordinates that brings the metric into the form (22). We also give a more extensive discussion of the counting of non-trivial parameters in this example.

\section{2 $D=2 n$ dimensions}

In this case, in addition to performing the Wick rotation of the radial variable as in (15), one must additionally rescale the mass by a factor of $\mathrm{i}$ in order to obtain a real metric. We then define functions

$$
U_{\mu}=\prod_{\nu=1}^{\prime n}\left(x_{\nu}^{2}-x_{\mu}^{2}\right), \quad X_{\mu}=-\left(1-g^{2} x_{\mu}^{2}\right) \prod_{k=1}^{n-1}\left(a_{k}^{2}-x_{\mu}^{2}\right)-2 M_{\mu} x_{\mu}
$$




$$
\widetilde{W}=\prod_{\nu=1}^{n}\left(1-g^{2} x_{\nu}^{2}\right), \quad \tilde{\gamma}_{i}=\prod_{\nu=1}^{n}\left(a_{i}^{2}-x_{\nu}^{2}\right)
$$

where $M_{n}=\mathrm{i} M$ and $M_{\alpha}=L_{\alpha}$. The even-dimensional Kerr-NUT-AdS metrics (13) can then be written as

$$
d s^{2}=\sum_{\mu=1}^{n}\left\{\frac{U_{\mu}}{X_{\mu}} d x_{\mu}^{2}+\frac{X_{\mu}}{U_{\mu}}\left[\frac{\widetilde{W}}{1-g^{2} x_{\mu}^{2}} d \tilde{t}-\sum_{i=1}^{n-1} \frac{\tilde{\gamma}_{i}}{a_{i}^{2}-x_{\mu}^{2}} d \tilde{\phi}_{i}\right]^{2}\right\} .
$$

We find that the inverse of the metric (28) is given by

$$
\left(\frac{\partial}{\partial s}\right)^{2}=\sum_{\mu=1}^{n}\left\{\frac{X_{\mu}}{U_{\mu}}\left(\frac{\partial}{\partial x_{\mu}}\right)^{2}+\frac{S_{\mu}}{U_{\mu} X_{\mu}}\left[\frac{1}{\left(\prod_{k} \Xi_{k}\right)} \frac{\partial}{\partial \tilde{t}}+\sum_{k=1}^{n-1} \frac{1-g^{2} x_{\mu}^{2}}{\Xi_{k} B_{k}\left(a_{k}^{2}-x_{\mu}^{2}\right)} \frac{\partial}{\partial \tilde{\phi}_{k}}\right]^{2}\right\},
$$

where

$$
S_{\mu}=\prod_{k=1}^{n-1}\left(a_{k}^{2}-x_{\mu}^{2}\right)^{2}, \quad B_{j}=\prod_{k=1}^{\prime n-1}\left(a_{j}^{2}-a_{k}^{2}\right) .
$$

Note that in terms of the original canonically-normalised coordinates $t$ and $\phi_{i}$, the inverse metric (29) takes the slightly simpler form

$$
\left(\frac{\partial}{\partial s}\right)^{2}=\sum_{\mu=1}^{n}\left\{\frac{X_{\mu}}{U_{\mu}}\left(\frac{\partial}{\partial x_{\mu}}\right)^{2}+\frac{S_{\mu}}{U_{\mu} X_{\mu}}\left[\frac{\partial}{\partial t}+\sum_{k=1}^{n-1} \frac{a_{k}\left(1-g^{2} x_{\mu}^{2}\right)}{\left(a_{k}^{2}-x_{\mu}^{2}\right)} \frac{\partial}{\partial \phi_{k}}\right]^{2}\right\},
$$

The even-dimensional Kerr-NUT-AdS metrics (13) and (28) also have a set of discrete symmetries under which any one of the rotation parameters $a_{i}$ is inverted through the AdS radius $1 / g$. Thus, for example, $(28)$ is invariant under the set of transformations

$$
\begin{aligned}
& a_{1} g \rightarrow \frac{1}{a_{1} g}, \quad a_{j} \rightarrow \frac{a_{j}}{a_{1} g}, \quad 2 \leq j \leq n-1, \\
& M_{\mu} \rightarrow \frac{M_{\mu}}{\left(a_{1} g\right)^{2 n-1}}, \quad g t \rightarrow \phi_{1}, \quad \phi_{1} \rightarrow g t, \quad x_{\mu} \rightarrow \frac{x_{\mu}}{a_{1} g},
\end{aligned}
$$

with $\phi_{j}$ for $2 \leq j \leq n-1$ left unchanged. This, and the other permutation-related inversion symmetries, can always map a metric with over-rotation (one or more parameters $a_{i}$ satisfying $\left|a_{i} g\right|>1$ ) into a metric with under-rotation (all parameters satisfying $\left|a_{i} g\right|<1$ ).

Again, we find that the new Kerr-NUT-AdS metrics in dimension $D=2 n$, which we have obtained in (13) and (28), can be further simplified and written elegantly in a form that is a natural higher-dimensional analogue of the four-dimensional metrics in [9]. After making the Wick rotation of the radial variable, as in (15), we then find that after appropriate linear redefinitions of the time and azimuthal coordinates, the $D=2 n$ Kerr-NUT-AdS metrics can be written as

$$
d s^{2}=\sum_{\mu=1}^{n}\left\{\frac{d x_{\mu}^{2}}{Q_{\mu}}+Q_{\mu}\left(\sum_{k=0}^{n-1} A_{\mu}^{(k)} d \psi_{k}\right)^{2}\right\}
$$


where we define

$$
\begin{aligned}
Q_{\mu} & =\frac{X_{\mu}}{U_{\mu}}, \quad U_{\mu}=\prod_{\nu=1}^{\prime n}\left(x_{\nu}^{2}-x_{\mu}^{2}\right), \quad X_{\mu}=\sum_{k=0}^{n} c_{k} x_{\mu}^{2 k}+2 b_{\mu} x_{\mu}, \\
A_{\mu}^{(k)} & =\sum_{\nu_{1}<\nu_{2}<\cdots<\nu_{k}}^{\prime} x_{\nu_{1}}^{2} x_{\nu_{2}}^{2} \cdots x_{\nu_{k}}^{2} .
\end{aligned}
$$

Again, the prime on the summation symbol in the definition of $A_{\mu}^{k}$ indicates that the index value $\mu$ is omitted in the summations of the $\nu$ indices over the range $[1, n]$. The constants $c_{k}$ and $b_{\mu}$ are arbitrary, with $c_{n}=(-1)^{n+1} g^{2}$ determining the value of the cosmological constant, $R_{\mu \nu}=-(2 n-1) g^{2} g_{\mu \nu}$. The remaining constants $c_{k}$ and $b_{\mu}$ are related to the rotation parameters, mass and NUT parameters in the obvious way that follows by comparing $X_{\mu}$ in (34) with $X_{\mu}$ in (27).

In this even-dimensional case there is ostensibly a mismatch between the total number of parameters in the metrics (13) or (28), namely $(n-1)$ rotation parameters $a_{i}$, the mass $M$ and the $(n-1)$ NUT parameters $L_{\alpha}$, and the number of parameters in the polynomials $X_{\mu}$, namely $n$ constants $c_{k}$ for $0 \leq k \leq n-1$, and $n$ constants $b_{\mu}$. However, there is also a scaling symmetry that leaves the metric (33) invariant, namely

$$
\begin{aligned}
& x_{\mu} \rightarrow \lambda x_{\mu}, \quad \psi_{k} \rightarrow \lambda^{-2 k-1} \psi_{k}, \\
& c_{k} \rightarrow \lambda^{2 n-2 k} c_{k}, \quad b_{\mu} \rightarrow \lambda^{2 n} b_{\mu} .
\end{aligned}
$$

This implies one parameter in $X_{\mu}$ is trivial, leaving $2 n-1$ non-trivial parameters in total in the general Kerr-NUT-AdS solution in dimension $D=2 n .^{3}$

It is useful also to record the inverse of the metric (33), which we find to be

$$
\left(\frac{\partial}{\partial s}\right)^{2}=\sum_{\mu=1}^{n}\left\{Q_{\mu}\left(\frac{\partial}{\partial x_{\mu}}\right)^{2}+\frac{1}{Q_{\mu} U_{\mu}^{2}}\left[\sum_{k=0}^{n-1}(-1)^{k} x_{\mu}^{2(n-1-k)} \frac{\partial}{\partial \psi_{k}}\right]^{2}\right\} .
$$

The specific case of the Kerr-NUT-AdS metric in $D=6$ dimensions is discussed in section 4.2, including the explicit transformation of the time and azimuthal coordinates that brings the metric into the form (33).

\footnotetext{
${ }^{3}$ It should be emphasised that there is a significant difference therefore between even and odd dimensions, as regards the number of non-trivial NUT charges that can be introduced. In even dimensions $D=2 n$ the general Kerr-AdS metrics can be augmented with the introduction of $(n-1)$ non-trivial NUT parameters, while in odd dimensions $D=2 n+1$ the general Kerr-AdS metrics can be augmented with the introduction of $(n-2)$ non-trivial NUT parameters. Thus, in particular, there is a non-trivial NUT charge in $D=4$, but there is no non-trivial NUT charge in $D=5$. In odd dimensions, only in $D=7$ and above does one have non-trivial NUT charges.
} 


\section{Kerr-NUT-AdS Metrics in $D=6$ and $D=7$}

\subsection{Seven-dimensional Kerr-NUT-AdS}

Here we present the specific example of $D=7$, with rotation parameters $a_{i}=\{a, b, c\}$, mass $M$ and two NUT parameters $L_{1}$ and $L_{2}$. The Kerr-NUT-AdS metric is given by

$$
\begin{aligned}
& d s^{2}= \frac{\left(r^{2}+y^{2}\right)\left(r^{2}+z^{2}\right) d r^{2}}{X}+\frac{\left(r^{2}+y^{2}\right)\left(y^{2}-z^{2}\right) d y^{2}}{Y}+\frac{\left(r^{2}+z^{2}\right)\left(z^{2}-y^{2}\right) d z^{2}}{Z} \\
&-\frac{X}{\left(r^{2}+y^{2}\right)\left(r^{2}+z^{2}\right)}\left[\left(1-g^{2} y^{2}\right)\left(1-g^{2} z^{2}\right) d \tilde{t}-a^{2}\left(a^{2}-y^{2}\right)\left(a^{2}-z^{2}\right) d \tilde{\phi}_{1}\right. \\
&\left.-b^{2}\left(b^{2}-y^{2}\right)\left(b^{2}-z^{2}\right) d \tilde{\phi}_{2}-c^{2}\left(c^{2}-y^{2}\right)\left(c^{2}-z^{2}\right) d \tilde{\phi}_{3}\right]^{2} \\
&+\frac{Y}{\left(r^{2}+y^{2}\right)\left(y^{2}-z^{2}\right)}\left[\left(1+g^{2} r^{2}\right)\left(1-g^{2} z^{2}\right) d \tilde{t}-a^{2}\left(a^{2}+r^{2}\right)\left(a^{2}-z^{2}\right) d \tilde{\phi}_{1}\right. \\
&+\frac{\left.Z-b^{2}\left(b^{2}+r^{2}\right)\left(b^{2}-z^{2}\right) d \tilde{\phi}_{2}-c^{2}\left(c^{2}+r^{2}\right)\left(c^{2}-z^{2}\right) d \tilde{\phi}_{3}\right]^{2}}{\left(r^{2}+z^{2}\right)\left(z^{2}-y^{2}\right)}\left[\left(1+g^{2} r^{2}\right)\left(1-g^{2} y^{2}\right) d \tilde{t}-a^{2}\left(a^{2}+r^{2}\right)\left(a^{2}-y^{2}\right) d \tilde{\phi}_{1}\right. \\
&\left.-b^{2}\left(b^{2}+r^{2}\right)\left(b^{2}-y^{2}\right) d \tilde{\phi}_{2}-c^{2}\left(c^{2}+r^{2}\right)\left(c^{2}-y^{2}\right) d \tilde{\phi}_{3}\right]^{2} \\
&+\frac{a^{2} b^{2} c^{2}}{r^{2} y^{2} z^{2}}\left[\left(1+g^{2} r^{2}\right)\left(1-g^{2} y^{2}\right)\left(1-g^{2} z^{2}\right) d \tilde{t}-\left(a^{2}+r^{2}\right)\left(a^{2}-y^{2}\right)\left(a^{2}-z^{2}\right) d \tilde{\phi}_{1}\right. \\
&\left.-\left(b^{2}+r^{2}\right)\left(b^{2}-y^{2}\right)\left(b^{2}-z^{2}\right) d \tilde{\phi}_{2}-\left(c^{2}+r^{2}\right)\left(c^{2}-y^{2}\right)\left(c^{2}-z^{2}\right) d \tilde{\phi}_{3}\right]^{2}, \quad
\end{aligned}
$$

where

$$
\begin{aligned}
& \tilde{t}=\frac{t}{\Xi_{a} \Xi_{b} \Xi_{c}}, \quad \tilde{\phi}_{1}=\frac{\phi_{1}}{a \Xi_{a}\left(b^{2}-a^{2}\right)\left(c^{2}-a^{2}\right)}, \\
& \tilde{\phi}_{2}=\frac{\phi_{2}}{b \Xi_{b}\left(a^{2}-b^{2}\right)\left(c^{2}-b^{2}\right)}, \quad \tilde{\phi}_{3}=\frac{\phi_{3}}{c \Xi_{c}\left(a^{2}-c^{2}\right)\left(b^{2}-c^{2}\right)}, \\
& \Xi_{a}=1-a^{2} g^{2}, \quad \Xi_{b}=1-b^{2} g^{2}, \quad \Xi_{c}=1-c^{2} g^{2}, \\
& X=\frac{1}{r^{2}}\left(1+g^{2} r^{2}\right)\left(a^{2}+r^{2}\right)\left(b^{2}+r^{2}\right)\left(c^{2}+r^{2}\right)-2 M, \\
& Y=\frac{1}{y^{2}}\left(1-g^{2} y^{2}\right)\left(a^{2}-y^{2}\right)\left(b^{2}-y^{2}\right)\left(c^{2}-y^{2}\right)+2 L_{1}, \\
& Z=\frac{1}{z^{2}}\left(1-g^{2} z^{2}\right)\left(a^{2}-z^{2}\right)\left(b^{2}-z^{2}\right)\left(c^{2}-z^{2}\right)+2 L_{2} .
\end{aligned}
$$

Note that regularity of the metric dictates that the coordinates $\phi_{i}$ each have period $2 \pi$ when the NUT parameters $L_{1}$ and $L_{2}$ are set to zero.

The metric has six parameters, $\left(a, b, c, M, L_{1}, L_{2}\right)$, but one of them is redundant. To show this, we first rewrite the metric after making the coordinate transformations

$$
t=t^{\prime}+\left(a^{2}+b^{2}+c^{2}\right) \psi_{1}+\left(a^{2} b^{2}+b^{2} c^{2}+c^{2} a^{2}\right) \psi_{2}+a^{2} b^{2} c^{2} \psi_{3},
$$




$$
\begin{aligned}
& \frac{\phi_{1}}{a}=\psi_{1}+\left(b^{2}+c^{2}\right) \psi_{2}+b^{2} c^{2} \psi_{3}+g^{2}\left(t^{\prime}+\left(b^{2}+c^{2}\right) \psi_{1}+b^{2} c^{2} \psi_{2}\right), \\
& \frac{\phi_{2}}{b}=\psi_{1}+\left(a^{2}+c^{2}\right) \psi_{2}+a^{2} c^{2} \psi_{3}+g^{2}\left(t^{\prime}+\left(a^{2}+c^{2}\right) \psi_{1}+a^{2} c^{2} \psi_{2}\right), \\
& \frac{\phi_{3}}{c}=\psi_{1}+\left(a^{2}+b^{2}\right) \psi_{2}+a^{2} b^{2} \psi_{3}+g^{2}\left(t^{\prime}+\left(a^{2}+b^{2}\right) \psi_{1}+a^{2} b^{2} \psi_{2}\right),
\end{aligned}
$$

which leads to

$$
\begin{aligned}
d s^{2}= & \frac{\left(r^{2}+y^{2}\right)\left(r^{2}+z^{2}\right) d r^{2}}{X}+\frac{\left(r^{2}+y^{2}\right)\left(y^{2}-z^{2}\right) d y^{2}}{Y}+\frac{\left(r^{2}+z^{2}\right)\left(z^{2}-y^{2}\right) d z^{2}}{Z} \\
& -\frac{X}{\left(r^{2}+y^{2}\right)\left(r^{2}+z^{2}\right)}\left(d t^{\prime}+\left(y^{2}+z^{2}\right) d \psi_{1}+y^{2} z^{2} d \psi_{2}\right)^{2} \\
& +\frac{Y}{\left(r^{2}+y^{2}\right)\left(z^{2}-y^{2}\right)}\left(d t^{\prime}+\left(z^{2}-r^{2}\right) d \psi_{1}-r^{2} z^{2} d \psi_{2}\right)^{2} \\
& +\frac{Z}{\left(r^{2}+z^{2}\right)\left(y^{2}-z^{2}\right)}\left(d t^{\prime}+\left(y^{2}-r^{2}\right) d \psi_{1}-r^{2} y^{2} d \psi_{2}\right)^{2} \\
& +\frac{C_{3}}{r^{2} y^{2} z^{2}}\left(d t^{\prime}+\left(y^{2}+z^{2}-r^{2}\right) d \psi_{1}+\left(y^{2} z^{2}-r^{2} y^{2}-r^{2} z^{2}\right) d \psi_{2}-r^{2} y^{2} z^{2} d \psi_{3}\right)^{2} .
\end{aligned}
$$

The functions $X, Y$ and $Z$ can be expressed as

$$
\begin{aligned}
& X=g^{2} r^{6}+C_{0} r^{4}+C_{1} r^{2}+C_{2}-2 M+\frac{C_{3}}{r^{2}} \\
& Y=g^{2} y^{6}-C_{0} y^{4}+C_{1} y^{2}-C_{2}+2 L_{1}+\frac{C_{3}}{y^{2}} \\
& Z=g^{2} z^{6}-C_{0} z^{4}+C_{1} z^{2}-C_{2}+2 L_{2}+\frac{C_{3}}{z^{2}}
\end{aligned}
$$

where

$$
\begin{aligned}
& C_{0}=1+g^{2}\left(a^{2}+b^{2}+c^{2}\right), \quad C_{1}=a^{2}+b^{2}+c^{2}+g^{2}\left(a^{2} b^{2}+b^{2} c^{2}+c^{2} a^{2}\right), \\
& C_{2}=a^{2} b^{2}+b^{2} c^{2}+c^{2} a^{2}+g^{2} a^{2} b^{2} c^{2}, \quad C_{3}=a^{2} b^{2} c^{2} .
\end{aligned}
$$

We can now view the solution as being parameterised by $\left(C_{0}, C_{1}, C_{3}\right)$, together with $X_{0}=$ $C_{2}-2 M, Y_{0}=2 L_{1}-C_{2}, Z_{0}=2 L_{2}-C_{2}$. The solution has a scaling symmetry, namely

$$
\begin{aligned}
& r \rightarrow \lambda r, \quad y \rightarrow \lambda y, \quad z \rightarrow \lambda z, \\
& C_{0} \rightarrow \lambda^{2} C_{0}, \quad C_{1} \rightarrow \lambda^{4} C_{1}, \quad C_{3} \rightarrow \lambda^{8} C_{3}, \\
& X_{0} \rightarrow \lambda^{6} X_{0}, \quad Y_{0} \rightarrow \lambda^{6} Y_{0}, \quad Z_{0} \rightarrow \lambda^{6} Z_{0}, \\
& \tilde{t} \rightarrow \lambda^{-1} \tilde{t}, \quad \psi_{1} \rightarrow \lambda^{-3} \psi_{1}, \quad \psi_{2} \rightarrow \lambda^{-5} \psi_{2}, \quad \psi_{3} \rightarrow \lambda^{-7} \psi_{3},
\end{aligned}
$$

This implies that one of the parameters in (43) can be set to 1 without loss of generality. In turn, this allows us to set one of the original parameters, say $L_{2}$ to zero. Thus there are actually five non-trivial parameters in the solution. 
For any fixed gauged choice of $M, L_{1}, L_{2}$, the metric still has discrete residual symmetry, namely

$$
a \rightarrow \frac{1}{a g^{2}}, \quad b \rightarrow \frac{b}{a g}, \quad c \rightarrow \frac{c}{a g}, \quad\left\{M, L_{1}, L_{2}\right\} \rightarrow \lambda^{6}\left\{M, L_{1}, L_{2}\right\},
$$

with $\lambda=1 /(a g)$.

\subsection{Six-dimensional Kerr-NUT-AdS}

Here we present the explicit $D=6$ metric, given by

$$
\begin{aligned}
& d s^{2}= \frac{\left(r^{2}+y^{2}\right)\left(r^{2}+z^{2}\right) d r^{2}}{X}+\frac{\left(r^{2}+y^{2}\right)\left(y^{2}-z^{2}\right) d y^{2}}{Y}+\frac{\left(r^{2}+z^{2}\right)\left(z^{2}-y^{2}\right) d z^{2}}{Z} \\
&-\frac{X}{\left(r^{2}+y^{2}\right)\left(r^{2}+z^{2}\right)}\left(\left(1-g^{2} y^{2}\right)\left(1-g^{2} z^{2}\right) d \tilde{t}-\left(a^{2}-y^{2}\right)\left(a^{2}-z^{2}\right) d \tilde{\phi}_{1}\right. \\
&\left.-\left(b^{2}-y^{2}\right)\left(b^{2}-z^{2}\right) d \tilde{\phi}_{2}\right)^{2} \\
&+\frac{Y}{\left(r^{2}+y^{2}\right)\left(y^{2}-z^{2}\right)}\left(\left(1+g^{2} r^{2}\right)\left(1-g^{2} z^{2}\right) d \tilde{t}-\left(a^{2}+r^{2}\right)\left(a^{2}-z^{2}\right) d \tilde{\phi}_{1}\right. \\
&\left.-\left(b^{2}+r^{2}\right)\left(b^{2}-z^{2}\right) d \tilde{\phi}_{2}\right)^{2} \\
&+\frac{Z}{\left(r^{2}+z^{2}\right)\left(z^{2}-y^{2}\right)}\left(\left(1+g^{2} r^{2}\right)\left(1-g^{2} y^{2}\right) d \tilde{t}-\left(a^{2}+r^{2}\right)\left(a^{2}-y^{2}\right) d \tilde{\phi}_{1}\right. \\
&\left.-\left(b^{2}+r^{2}\right)\left(b^{2}-y^{2}\right) d \tilde{\phi}_{2}\right)^{2} .
\end{aligned}
$$

where $X, Y$ and $Z$ are given by

$$
\begin{aligned}
& X=\left(1+g^{2} r^{2}\right)\left(r^{2}+a^{2}\right)\left(r^{2}+b^{2}\right)-2 M r, \quad Y=-\left(1-g^{2} y^{2}\right)\left(a^{2}-y^{2}\right)\left(b^{2}-y^{2}\right)-2 L_{1} y \\
& Z=-\left(1-g^{2} z^{2}\right)\left(a^{2}-z^{2}\right)\left(b^{2}-z^{2}\right)-2 L_{2} z .
\end{aligned}
$$

The coordinate $\tilde{t}, \tilde{\phi}_{1}$ and $\tilde{\phi}_{2}$ are related to the canonically defined $t, \phi_{1}$ and $\phi_{2}$ by (14). We can then make the coordinate transformation

$$
\begin{aligned}
t & =t^{\prime}+\left(a^{2}+b^{2}\right) \psi_{1}+a^{2} b^{2} \psi_{2}, \quad \frac{\phi_{1}}{a}=\psi_{1}+b^{2} \psi_{2}+g^{2}\left(d \tilde{t}+b^{2} \psi_{1}\right), \\
\frac{\phi_{2}}{b} & =\psi_{1}+a^{2} \psi_{2}+g^{2}\left(d \tilde{t}+a^{2} \psi_{1}\right)
\end{aligned}
$$

which leads to the metric

$$
\begin{aligned}
d s^{2}= & \frac{\left(r^{2}+y^{2}\right)\left(r^{2}+z^{2}\right) d r^{2}}{X}+\frac{\left(r^{2}+y^{2}\right)\left(y^{2}-z^{2}\right) d y^{2}}{Y}+\frac{\left(r^{2}+z^{2}\right)\left(z^{2}-y^{2}\right) d z^{2}}{Z} \\
& -\frac{X}{\left(r^{2}+y^{2}\right)\left(r^{2}+z^{2}\right)}\left(d t^{\prime}+\left(y^{2}+z^{2}\right) d \psi_{1}+y^{2} z^{2} d \psi_{2}\right)^{2} \\
& +\frac{Y}{\left(r^{2}+y^{2}\right)\left(y^{2}-z^{2}\right)}\left(d t^{\prime}+\left(z^{2}-r^{2}\right) d \psi_{1}-r^{2} z^{2} d \psi_{2}\right)^{2} \\
& +\frac{Z}{\left(r^{2}+z^{2}\right)\left(z^{2}-y^{2}\right)}\left(d t^{\prime}+\left(y^{2}-r^{2}\right) d \psi_{1}-r^{2} y^{2} d \psi_{2}\right)^{2} .
\end{aligned}
$$


The functions $X, Y$ and $Z$ given in (46) can now be written as

$$
\begin{aligned}
& X=g^{6} r^{6}+C_{0} r^{4}+C_{1} r^{2}-2 M r+C_{2}, \\
& Y=g^{6} y^{6}-C_{0} y^{4}+C_{1} y^{2}-2 L_{1} y-C_{2}, \\
& Z=g^{6} z^{6}-C_{0} z^{4}+C_{1} z^{2}-2 L_{1} z-C_{2},
\end{aligned}
$$

where $C_{i}$ are constants, expressed in terms two constants $a$ and $b$, given by

$$
C_{0}=1+g^{2}\left(a^{2}+b^{2}\right), \quad C_{1}=a^{2}+b^{2}+g^{2} a^{2} b^{2}, \quad C_{2}=a^{2} b^{2} .
$$

In fact, the constants $C_{i}$ can be arbitrary, since the form of the metric has the following symmetry:

$$
\begin{aligned}
& r \rightarrow \lambda r, \quad y \rightarrow \lambda y, \quad z \rightarrow \lambda z, \\
& C_{0} \rightarrow \lambda^{2} C_{0}, \quad C_{1} \rightarrow \lambda^{4} C_{1}, \quad C_{2} \rightarrow \lambda^{6} C_{2}, \\
& M \rightarrow \lambda^{5} M, \quad L_{1} \rightarrow \lambda^{5} L_{1}, \quad L_{2} \rightarrow \lambda^{5} L_{2}, \\
& \tilde{t} \rightarrow \lambda^{-1} \tilde{t}, \quad \psi_{1} \rightarrow \lambda^{-3} \psi_{1}, \quad \psi_{2} \rightarrow \lambda^{-5} \psi_{2} .
\end{aligned}
$$

Thus to fix $C_{i}$ as given by (49) is to have fixed the symmetry. It follows that unlike in the case of odd dimensions, the NUT parameters here are all non-trivial. For the above fixed parameter gauge, the metric has residual discrete symmetry, namely

$$
a \rightarrow \frac{1}{a g^{2}}, \quad b \rightarrow \frac{b}{a g}
$$

with $\lambda=a g$.

The form in which the six-dimensional Kerr-NUT-AdS metric is written in equation (48) is closely analogous to the form of the four-dimensional Plebanski metrics [9].

\section{BPS Limits}

\subsection{BPS limit for $D=2 n+1$}

In this section we shall investigate the BPS limit of the odd-dimensional Kerr-NUT-AdS metrics. In this limit the metrics admit Killing spinors, and if one furthermore performs a Euclideanisation to positive-definite metric signature, and sets the cosmological constant to be positive (by taking $g^{2}$ to be negative) one will obtain Einstein-Sasaki metrics.

For convenience, we shall scale the metrics in this limit so that their Ricci tensor is the same as that of a unit sphere of the same dimension. This is achieved by setting $g=\mathrm{i}$. It is 
convenient also to write the metrics in a specific asymptotically-rotating frame, by sending $\phi_{i} \rightarrow \phi_{i}-g d t$.

We shall first consider the 7-dimensional metric discussed in the previous section. The Euclideanisation is achieved by sending

$$
t \rightarrow \mathrm{i} \tau, \quad a \rightarrow \mathrm{i} a, \quad b \rightarrow \mathrm{i} b, \quad c \rightarrow \mathrm{i} c .
$$

To take the BPS limit we define

$$
\begin{aligned}
& 1-a^{2}=\alpha \epsilon, \quad 1-b^{2}=\beta \epsilon, \quad 1-c^{2}=\gamma \epsilon, \\
& 1-r^{2}=x \epsilon, \quad 1+y^{2} \rightarrow y \epsilon, \quad 1+z^{2} \rightarrow z \epsilon, \\
& M=m \epsilon^{4}, \quad L_{1}=\ell_{1} \epsilon^{4}, \quad L_{2}=\ell_{2} \epsilon^{4},
\end{aligned}
$$

and then send $\epsilon \rightarrow 0$. This leads to the metric

$$
d s_{7}^{2}=(d \tau+\mathcal{A})^{2}+d s_{6}^{2},
$$

where

$$
\begin{aligned}
d s_{6}^{2}= & \frac{(x-y)(x-z) d x^{2}}{4 X}+\frac{(y-x)(y-z) d y^{2}}{4 Y}+\frac{(z-x)(z-y)}{4 Z} d z^{2} \\
& +\frac{X}{(x-y)(x-z)}\left((\alpha-y)(\alpha-z) d \tilde{\phi}_{1}+(\beta-y)(\beta-z) d \tilde{\phi}_{2}+(\gamma-y)(\gamma-z) d \tilde{\phi}_{3}\right)^{2} \\
& +\frac{Y}{(y-x)(y-z)}\left((\alpha-x)(\alpha-z) d \tilde{\phi}_{1}+(\beta-x)(\beta-z) d \tilde{\phi}_{2}+(\gamma-x)(\gamma-z) d \tilde{\phi}_{3}\right)^{2} \\
& +\frac{Z}{(z-x)(z-y)}\left((\alpha-x)(\alpha-y) d \tilde{\phi}_{1}+(\beta-x)(\beta-y) d \tilde{\phi}_{2}+(\gamma-x)(\gamma-y) d \tilde{\phi}_{3}\right)^{2} \\
\mathcal{A}= & (\alpha-x)(\alpha-y)(\alpha-z) d \tilde{\phi}_{1}+(\beta-x)(\beta-y)(\beta-z) d \tilde{\phi}_{1}+(\gamma-x)(\gamma-y)(\gamma-z) d \tilde{\phi}_{1} \\
X= & x(\alpha-x)(\beta-x)(\gamma-x)-2 m, \quad Y=y(\alpha-y)(\beta-y)(\gamma-y)-2 \ell_{1}, \\
Z= & z(\alpha-z)(\beta-z)(\gamma-z)-2 \ell_{2} .
\end{aligned}
$$

The $\tilde{\phi}_{i}$ are related to the original $\phi_{i}$ by the constant scalings

$$
\begin{aligned}
& \phi_{1}=\alpha(\alpha-\beta)(\alpha-\gamma) \tilde{\phi}_{1}, \quad \phi_{2}=\beta(\beta-\alpha)(\beta-\gamma) \tilde{\phi}_{2}, \\
& \phi_{3}=\gamma(\gamma-\alpha)(\gamma-\beta) \tilde{\phi}_{3} .
\end{aligned}
$$

For the general case of $D=2 n+1$ dimensions, we find after performing analogous computations that the Einstein-Sasaki metric is given by

$$
d s_{2 n+1}^{2}=(d \tau+\mathcal{A})^{2}+d s_{2 n}^{2}
$$


where

$$
\begin{aligned}
d s_{2 n}^{2} & =\sum_{\mu=1}^{n} \frac{U_{\mu} d x_{\mu}^{2}}{4 X_{\mu}}+\sum_{\mu=1}^{n} \frac{X_{\mu}}{U_{\mu}}\left(\sum_{i=1}^{n} \frac{W_{i} d \tilde{\phi}_{i}}{\alpha_{i}-x_{\mu}}\right)^{2}, \\
\mathcal{A} & =\sum_{i=1}^{n} W_{i} d \tilde{\phi}_{i}, \quad U_{\mu}=\prod_{\nu=1}^{\prime n}\left(x_{\nu}-x_{\mu}\right), \\
X_{\mu} & =x_{\mu} \prod_{i=1}^{n}\left(\alpha_{i}-x_{\mu}\right)-2 \ell_{\mu}, \quad W_{i}=\prod_{\nu=1}^{n}\left(\alpha_{i}-x_{\nu}\right)
\end{aligned}
$$

Thus we obtain a large class of local Einstein-Sasaki metrics in arbitrary $(2 n+1)$ dimensions. These metrics extend the results obtained in $[10,11]$, where there were no NUT charges, and those in $[12,6]$, where metrics of cohomogeneity two were considered. We expect that $(57,58)$ is the most general metric for Einstein-Sasaki spaces with $U(1)^{n+1}$ isometry in $(2 n+1)$ dimensions.

It is of considerable interest to study the global structure of these Einstein-Sasaki metrics, and thereby to obtain the conditions on the parameters under which they extend onto smooth manifolds. This was done for $D=5$ in $[10,11]$, where complete metrics for the Einstein-Sasaki manifolds $L^{p q r}$ were obtained. Those results extended previous results for the $Y^{p q}[13]$ manifolds, which corresponded to the specialisation where the two angular momentum parameters were set equal. For seven dimensions, the global structure has been previously discussed for various special cases. When $Y$ and $Z$ in (55) both have a double root, the solution reduces to that obtained in [14], where the global structure was analysed in detail. If two angular momenta are set equal, the solution reduces to that obtained in [12] where the global structure was also discussed. Aside from these special cases, our general results in $D=7$ that we have obtained in this paper are new. Similarly, our results in $D \geq 9$ extend those obtained previously.

\subsection{BPS limit for $D=2 n$}

The BPS limit in this case can give rise to Ricci-flat Kähler metrics. Consider first the example of the six-dimensional Kerr-NUT-AdS metric. We perform a Euclideanisation and take an analogous BPS limit to the one we discussed above for the seven-dimensional case, by setting

$$
M=m \epsilon^{3}, \quad L_{1}=i \ell_{1} \epsilon^{3}, \quad L_{2}=i \ell_{2} \epsilon^{3} .
$$

In the BPS limit, when $\epsilon$ goes to zero, we obtain the Ricci flat metric

$$
d s_{6}^{2}=\frac{(y-x)(z-x) d x^{2}}{4 X}+\frac{(x-y)(z-y) d y^{2}}{4 Y}+\frac{(x-z)(y-z)}{4 Z} d z^{2}
$$




$$
\begin{aligned}
& +\frac{X}{(y-x)(z-x)}\left(y z d \tilde{\tau}-(\alpha-y)(\alpha-z) d \tilde{\phi}_{1}-(\beta-y)(\beta-z) d \tilde{\phi}_{2}\right)^{2} \\
& +\frac{Y}{(x-y)(z-y)}\left(x z d \tilde{\tau}-(\alpha-x)(\alpha-z) d \tilde{\phi}_{1}-(\beta-x)(\beta-z) d \tilde{\phi}_{2}\right)^{2} \\
& +\frac{Z}{(x-z)(y-z)}\left(x y d \tilde{\tau}-(\alpha-x)(\alpha-y) d \tilde{\phi}_{1}-(\beta-x)(\beta-y) d \tilde{\phi}_{2}\right)^{2} \\
X= & x(\alpha-x)(\beta-x)-2 m, \quad Y=y(\alpha-y)(\beta-y)-2 \ell_{1}, \\
Z= & z(\alpha-z)(\beta-z)-2 \ell_{2} .
\end{aligned}
$$

The coordinates $\tilde{\tau}$ and $\tilde{\phi}_{i}$ are related to the original $\tau$ and $\phi_{i}$ by the constant scalings

$$
\phi_{1}=\alpha(\alpha-\beta) \tilde{\phi}_{1}, \quad \phi_{2}=\beta(\beta-\alpha) \tilde{\phi}_{2}, \quad \tau=\alpha \beta \tilde{\tau}
$$

Note that this metric can in fact be viewed as the zero cosmological constant limit of the six-dimensional Einstein-Kähler metric (55) that we obtained above.

For the general case of $D=2 n$ dimensions, we find that the BPS limit of the Euclideanised Kerr-NUT-AdS metrics yields the Ricci-flat metrics

$$
\begin{aligned}
d s_{2 n}^{2} & =\sum_{\mu=1}^{n} \frac{U_{\mu} d x_{\mu}^{2}}{4 X_{\mu}}+\sum_{\mu=1}^{n} \frac{X_{\mu}}{U_{\mu}}\left(\frac{\gamma}{x_{\mu}} d \tilde{\tau}-\sum_{i=1}^{n-1} \frac{W_{i} d \tilde{\phi}_{i}}{\alpha_{i}-x_{\mu}}\right)^{2} \\
X_{\mu} & =x_{\mu} \prod_{i=1}^{n-1}\left(\alpha_{i}-x_{\mu}\right)-2 \ell_{\mu}, \quad U_{\mu}=\prod_{\nu=1}^{\prime n}\left(x_{\nu}-x_{\mu}\right) \\
W_{i} & =\prod_{\nu=1}^{n}\left(\alpha_{i}-x_{\nu}\right), \quad \gamma=\prod_{\nu=1}^{n} x_{\nu}
\end{aligned}
$$

Again, these metrics can be obtained also as limiting cases of the metrics (58), in which the cosmological constant is sent to zero.

\section{Conclusions}

In this paper, we have obtained new results for the inclusion of NUT parameters in the Kerr-AdS metrics that were constructed in [4, 5]. Our strategy for doing this involved first making a judicious choice of coordinates parameterising the latitude variables in the Kerr-AdS metrics. By making a change of variables analogous to one considered long ago by Jacobi in the theory of constrained dynamical systems, we were able to rewrite the Kerr-AdS solutions of $[4,5]$ in such a way that the metrics become diagonal in a set of unconstrained latitude coordinates $y_{\alpha}$. These coordinates then appear in a manner that closely parallels that of the radial variable $r$, and this immediately suggests a natural generalisation of the Kerr-AdS metrics to include NUT charges. We explicitly verified that the generalised 
metrics solve the Einstein equations in all dimensions $D \leq 15$, and we expect that they likewise solve the equations in all higher dimensions. After further changes of variable, we arrived at the very simple expressions (22) and (33) for the general Kerr-NUT-AdS metrics in all odd and even dimensions. These expressions can be thought of as natural generalisations of the four-dimensional results obtained in [9].

The general Kerr-NUT-AdS metrics that we have obtained in this paper have a total of $(2 n-1)$ non-trivial parameters, where the spacetime dimension is $D=2 n+1$ in the odd-dimensional case, and $D=2 n$ in the even-dimensional case. In odd dimensions these parameters can be viewed as comprising $n$ rotations, a mass, and $(n-2)$ NUT charges. In even dimensions they instead comprise $(n-1)$ rotations, a mass, and $(n-1)$ NUT charges. In odd dimensions, but not in even dimensions, there is some measure of arbitrariness in the interpretation of parameters as rotations or NUT charges.

An interesting feature of the Kerr-AdS and Kerr-NUT-AdS metrics that is uncovered by our work is that in all dimensions there exist discrete symmetries of the metrics in which one of the rotation parameters is inverted through the AdS radius $1 / g$, together with appropriate scalings of the other rotation parameters, the mass and the NUT charges. An implication of these symmetries is that any metric with over-rotation, i.e. where one or more of the rotation parameters exceeds the AdS radius, is identical, up to coordinate transformations, to a metric with only under-rotation. This was observed previously for the Kerr-AdS metric in $D=5$ [6]. The inversion symmetry was apparently not previously noticed in the four-dimensional Kerr-AdS metric, and we have presented it explicitly, in the standard coordinate system, in appendix B.

We also considered the BPS, or supersymmetric, limits of the Kerr-NUT-AdS metrics. In odd dimensions these yield, after Euclideanisation, new examples of Einstein-Sasaki metrics. We expect that by making appropriate choices for the various parameters in the solutions, one can obtain new examples of complete Einstein-Sasaki spaces defined on non-singular compact manifolds.

\section{Acknowledgement}

We are grateful to Gary Gibbons and Malcolm Perry for useful discussions. C.N.P. is grateful to the Relativity and Cosmology group at the C.M.S., Cambridge, for hospitality during part of this work. 


\section{A A symmetry between the time and azimuthal coordinates}

It can be observed from the expressions for the Kerr-NUT-AdS metrics that we obtained in section 3 that the time coordinate and the azimuthal angular coordinates appear on a very parallel footing. It is possible, therefore, to present further simplifications of the expressions (17) and (28) in odd and even dimensions that exploit this observation.

For the Kerr-NUT-AdS metrics in odd dimensions $D=2 n+1$, we make the definitions

$$
\begin{aligned}
& a_{0}=\frac{1}{g}, \quad \Gamma_{I}=\prod_{\nu=1}^{n}\left(a_{I}^{2}-x_{\nu}^{2}\right), \quad 0 \leq I \leq n, \\
& \tilde{\phi}_{0}=-g^{2 n} \tilde{t}, \quad X_{\mu}=\frac{g^{2}}{x_{\mu}^{2}} \prod_{I=0}^{n}\left(a_{I}^{2}-x_{\mu}^{2}\right)+2 M_{\mu} .
\end{aligned}
$$

The metric (17) can then be written as

$$
d s^{2}=\sum_{\mu=1}^{n}\left\{\frac{U_{\mu}}{X_{\mu}} d x_{\mu}^{2}+\frac{X_{\mu}}{U_{\mu}}\left(\sum_{I=0}^{n} \frac{a_{I}^{2} \Gamma_{I} d \tilde{\phi}_{I}}{a_{I}^{2}-x_{\mu}^{2}}\right)^{2}\right\}-\frac{\left(\prod_{k=1}^{n} a_{k}^{2}\right)}{\left(\prod_{\mu=1}^{n} x_{\mu}^{2}\right)}\left(\sum_{I=0}^{n} \Gamma_{I} d \tilde{\phi}_{I}\right)^{2} .
$$

For the Kerr-NUT-AdS metrics in even dimensions $D=2 n$, we make the definitions

$$
\begin{aligned}
& a_{0}=\frac{1}{g}, \quad \Gamma_{I}=\prod_{\nu=1}^{n}\left(a_{I}^{2}-x_{\nu}^{2}\right), \quad 0 \leq I \leq n-1, \\
& \tilde{\phi}_{0}=-g^{2 n-2} \tilde{t}, \quad X_{\mu}=-g^{2} \prod_{I=0}^{n-1}\left(a_{I}^{2}-x_{\mu}^{2}\right)-2 M_{\mu} x_{\mu} .
\end{aligned}
$$

The metric (28) can then be written as

$$
d s^{2}=\sum_{\mu=1}^{n}\left\{\frac{U_{\mu}}{X_{\mu}} d x_{\mu}^{2}+\frac{X_{\mu}}{U_{\mu}}\left(\sum_{I=0}^{n-1} \frac{\Gamma_{I} d \tilde{\phi}_{I}}{a_{I}^{2}-x_{\mu}^{2}}\right)^{2}\right\}
$$

\section{B Inversion symmetry of the $D=4$ rotating black hole}

We made the observation $n$ section 3 that there exists an inversion symmetry in all the KerrNUT-AdS metrics, in which one of the rotation parameters is inverted through the AdS radius, together with corresponding scalings of the other parameters. A case of particular interest is in four dimensions. (The inversion symmetry for the five-dimensional Kerr-AdS metric was given in [6].) The four-dimensional Kerr-AdS metric can be written as

$$
\begin{aligned}
d s^{2} & =\frac{\rho^{2}}{\Delta_{r}} d r^{2}+\frac{\rho^{2}}{\Delta_{\theta}} d \theta^{2}-\frac{\Delta_{r}}{\rho^{2}}\left(d t-\frac{a}{\Xi} \sin ^{2} \theta d \phi^{2}\right)^{2}+\frac{\Delta_{\theta} \sin ^{2} \theta}{\rho^{2}}\left(a d t-\frac{r^{2}+a^{2}}{\Xi} d \phi\right)^{2}, \\
\rho^{2} & =r^{2}+a^{2} \cos ^{2} \theta, \quad \Delta_{r}=\left(1+g^{2} r^{2}\right)\left(r^{2}+a^{2}\right)-2 M r, \quad \Delta_{\theta}=1-a^{2} g^{2} \cos ^{2} \theta,(67)
\end{aligned}
$$


where $\Xi=1-a^{2} g^{2}$. It is straightforward to verify that the metric is invariant under the transformation

$$
\begin{aligned}
& a \rightarrow \frac{1}{a g^{2}}, \quad M \rightarrow \frac{M}{a^{3} g^{3}}, \\
& r \rightarrow \frac{r}{a g}, \quad \cos \theta \rightarrow a g \cos \theta, \quad \phi \rightarrow-\frac{\phi}{a g}, \quad t \rightarrow a g t+\frac{\phi}{g} .
\end{aligned}
$$

Note that the metric (67) is written in a frame that is asymptotically rotating at infinity. As a consequence the required ignorable coordinate transformations in (68) that bring the transformed metric back to its original form do not, unlike those given in (32) for an asymptotically-static frame, simply involve an exchange of the azimuthal coordinate and $g$ times the time coordinate. If we define an asymptotically-static frame by replacing the

azimuthal coordinate with $\hat{\phi}=\phi+a g^{2} t$, then the last two transformations in (68) become simply

$$
\hat{\phi} \rightarrow g t, \quad g t \rightarrow \hat{\phi} .
$$

\section{References}

[1] J.F. Plebanski and M. Demianski, Rotating, charged, and uniformly accelerating mass in general relativity, Annals Phys. 98 (1976) 98.

[2] R.C. Myers and M.J. Perry, Black holes in higher dimensional space-times, Annals Phys. 172, 304 (1986).

[3] S.W. Hawking, C.J. Hunter and M.M. Taylor-Robinson, Rotation and the AdS/CFT correspondence, Phys. Rev. D59, 064005 (1999), hep-th/9811056.

[4] G.W. Gibbons, H. Lü, D.N. Page and C.N. Pope, The general Kerr-de Sitter metrics in all dimensions, J. Geom. Phys. 53, 49 (2005), hep-th/0404008.

[5] G.W. Gibbons, H. Lü, D.N. Page and C.N. Pope, Rotating black holes in higher dimensions with a cosmological constant, Phys. Rev. Lett. 93, 171102 (2004), hep-th/0409155.

[6] W. Chen, H. Lü and C.N. Pope, Kerr-de Sitter black holes with NUT charges, hepth/0601002.

[7] R.B. Mann and C. Stelea, New multiply nutty spacetimes, Phys. Lett. B 634, 448 (2006) hep-th/0508203.

[8] C. Jacobi, Vorlesungen über Dynamik, Reimer, Berlin (1866). 
[9] J.F. Plebanski, A class of solutions of Einstein-Maxwell equations, Ann. Phys. 90, 196 (1975).

[10] M. Cvetič, H.Lü, D.N. Page and C.N. Pope, New Einstein-Sasaki spaces in five and higher dimensions, Phys. Rev. Lett. 95, 071101 (2005), hep-th/0504225.

[11] M. Cvetič, H. Lü, D.N. Page and C.N. Pope, New Einstein-Sasaki and Einstein spaces from Kerr-de Sitter, hep-th/0505223.

[12] H. Lü, C.N. Pope and J.F. Vázquez-Poritz, A new construction of Einstein-Sasaki metrics in $D \geq 7$, hep-th/0512306.

[13] J.P. Gauntlett, D. Martelli, J. Sparks and D. Waldram, Sasaki-Einstein metrics on $S^{2} \times S^{3}$ Adv. Theor. Math. Phys. 8, 711 (2004), hep-th/0403002.

[14] W. Chen, H. Lü, C.N. Pope and J.F. Vázquez-Poritz, A note on Einstein-Sasaki metrics in $D \geq 7$, Class. Quant. Grav. 22, 3421 (2005), hep-th/0411218. 